Pacific

Journal of

Mathematics

A NOTE ON A FOURTH ORDER PDE WITH CRITICAL NONLINEARITY

Changyou Wang

Volume $216 \quad$ No. 2

October 2004 


\title{
A NOTE ON A FOURTH ORDER PDE WITH CRITICAL NONLINEARITY
}

\author{
Changyou Wang
}

\begin{abstract}
We consider the Euler-Lagrange equation of a functional arising from conformal geometry in four dimensions, a fourth order equation with borderline nonlinearity. We present a short proof of the fact that any $W^{2,2}$-solution is smooth.
\end{abstract}

\section{Introduction}

Let $(M, g)$ be a four-dimensional compact Riemannian manifold. Motivated by problems in four-dimensional spectral theory and conformal geometry, Chang and Yang $[\mathbf{C Y}]$ (cf. also Chang $[\mathbf{C}]$ ) introduced the functional $F$ : $W^{2,2}(M) \rightarrow \mathbf{R}:$

$$
F(w)=\int_{M}\left\{(\Delta w)^{2}+\left(\alpha \Delta w+\beta|D w|^{2}\right)^{2}+T(D w, D w)+E(w-\bar{w})\right\} d v,
$$

where $\alpha, \beta \in \mathbf{R}, \bar{w}=\frac{1}{\operatorname{vol} M} \int w ; E: \mathbf{R} \rightarrow \mathbf{R}$ and $T \in \operatorname{sym}^{2}\left(T^{*} M\right)$ satisfy:

$$
\max \left\{|E(x)|,\left|E^{\prime}(x)\right|\right\} \leq c_{1} e^{c_{2}|x|}, \quad|T(v, v)| \leq c_{3}|v|^{2} .
$$

Direct computations show that the Euler-Lagrange equation associated with critical points of $F$ on $W^{2,2}(M)$ is

$$
\begin{aligned}
2\left(1+\alpha^{2}\right) \Delta^{2} w+2 \beta \operatorname{div} & \left(\alpha D\left(|D w|^{2}\right)-\left(\alpha \Delta w+\beta|D w|^{2}\right) D w\right) \\
= & \operatorname{div}(T(D w, \cdot))-\left(E^{\prime}(w-\bar{w})-\bar{E}^{\prime}(w-\bar{w})\right)
\end{aligned}
$$

where $\bar{E}^{\prime}(w-\bar{w})=\frac{1}{\operatorname{vol} M} \int E^{\prime}(w-\bar{w})$. Chang, Gursky and Yang [CGY] proved that any $F$-minimizing solution $u \in W^{2,2}(M)$ to (1.3) is actually smooth. It was asked in [CGY] whether any weak solution $u \in W^{2,2}(M)$ is smooth. Indeed, Uhlenbeck and Viaclovsky [UV] confirmed this recently and proved the smoothness for any weak solution $u \in W^{2,2}(M)$ to (1.3). The proof in $[\mathbf{C G Y}]$ relied on $F$-minimality. The idea in $[\mathbf{U V}]$ is based on some uniqueness properties for small perturbations of $\Delta^{2}$ in various Sobolev spaces and seems to be an indirect argument. Here we provide an alternative and direct proof of the smoothness for weak solutions to (1.3); namely, we show that under a smallness assumption on the $W^{2,2}$ norm, the normalized $L^{p_{-}}$ norm of the gradient of $u$ on a ball decays like a positive power of the radius 
of the ball. This, combined with Morrey's decay lemma and the conformal invariance of the $W^{2,2}$ norm in dimension four, implies the Hölder continuity of $u$. Higher-order regularity then follows from [CGY]. This type of socalled $\epsilon_{0}$-decay lemma is very common in the context of regularity theory for harmonic maps (cf. Schoen-Uhlenbeck [SU]). In fact, this kind of idea was also employed by Chang, Wang and Yang in their study of the regularity problem of biharmonic maps into spheres [CWY].

Since regularity is a local result, we assume, for simplicity, that $M=\Omega \subset$ $\mathbf{R}^{4}$ is a bounded smooth domain, with the Euclidean metric $g$. Now we state the decay lemma:

Lemma A. There exist $\epsilon_{0}>0$ and $\theta_{0} \in\left(0, \frac{1}{2}\right)$ such that if $u \in W^{2,2}(\Omega)$ is a weak solution to (1.3) and if for $B_{r}(x) \subset \Omega$ we have

$$
\int_{B_{r}(x)}|D u|^{4}+\left|D^{2} u\right|^{2} \leq \epsilon_{0}^{2}
$$

then, for any $2<p<4$,

$$
\left(\theta_{0} r\right)^{p-4} \int_{B_{\theta_{0}}(x)}|D u|^{p} \leq \frac{1}{2} r^{p-4} \int_{B_{r}(x)}|D u|^{p}+C\left(p,\left\|D^{2} u\right\|_{L^{2}(\Omega)}\right) r^{p} .
$$

Since $u \in W^{2,2}(\Omega)$, the absolute continuity of $\int|D u|^{4}+\left|D^{2} u\right|^{2}$ implies that there exists an $r_{0}>0$ such that (1.4) holds for $u$ over any ball $B_{r}(x) \subset$ $\Omega$ with $0<r \leq r_{0}$. Therefore, we can apply the lemma repeatedly and conclude that there exists a $\delta_{0} \in(0,1)$ such that $r^{p-4} \int_{B_{r}(x)}|D u|^{p}$ behaves like $r^{p \delta_{0}}$ for all $0<r<r_{0}$ and $x \in \Omega$. This, combined with Morrey's lemma, implies that $u \in C^{\delta_{0}}(\Omega)$ and hence $u \in C^{\infty}(\Omega)$, via [CGY]. In particular, one has (cf. also $[\mathbf{U V}])$ :

Theorem B. If $u \in W^{2,2}(M)$ is a weak solution to (1.3), then $u \in C^{\infty}(M)$.

\section{Proof of Lemma A}

It follows from Fubini's theorem that there is an $s \in\left[\frac{r}{2}, r\right]$ such that

$$
\int_{\partial B_{s}(x)}|D u|^{4}+\left|D^{2} u\right|^{2} \leq 2 r^{-1} \int_{B_{r}(x)}|D u|^{4}+\left|D^{2} u\right|^{2} .
$$

Let $u_{1} \in W^{2,2}\left(B_{s}(x)\right)$ satisfy

$$
\begin{aligned}
\Delta^{2} u_{1} & =-\frac{\beta}{1+\alpha^{2}} \operatorname{div}\left(\alpha D\left(|D u|^{2}\right)-\left(\alpha \Delta u+\beta|D u|^{2}\right) D u\right), \\
u_{1} & =\frac{\partial u_{1}}{\partial r}=0 \quad \text { on } \partial B_{s}(x) .
\end{aligned}
$$


Let $u_{2} \in W^{2,2}\left(B_{s}(x)\right)$ satisfy

$$
\begin{aligned}
\Delta^{2} u_{2} & =\frac{1}{2\left(1+\alpha^{2}\right)}\left(\operatorname{div}(T(D u, \cdot))-\left(E^{\prime}(u-\bar{u})-\bar{E}^{\prime}(u-\bar{u})\right)\right), \\
u_{2} & =\frac{\partial u_{2}}{\partial r}=0 \quad \text { on } \partial B_{s}(x) .
\end{aligned}
$$

Let $u_{3}=u-u_{1}-u_{2} \in W^{2,2}\left(B_{s}(x)\right)$. Then we have

$$
\begin{array}{rlrl}
\Delta^{2} u_{3} & =0 & & \text { in } B_{s}(x), \\
u_{3}=u \quad \text { and } \quad \frac{\partial u_{3}}{\partial r}=\frac{\partial u}{\partial r} & & \text { on } \partial B_{s}(x) .
\end{array}
$$

For $u_{1}$, it follows (see, e.g., Lemma 2.2 of $[\mathbf{C W Y}]$ ) that for any $q \in\left(1, \frac{4}{3}\right)$

$$
\begin{aligned}
\left\|D^{3} u_{1}\right\|_{L^{q}\left(B_{s}(x)\right)} & \leq C\left\|\left|D u\left\|\left.D^{2} u|+| D u\right|^{2}|D u|\right\|_{L^{q}\left(B_{s}(x)\right)}\right.\right. \\
& \leq C\left(\left\|D^{2} u\right\|_{L^{2}\left(B_{s}(x)\right)}+\|D u\|_{L^{4}\left(B_{s}(x)\right)}^{2}\|D u\|_{L^{\frac{2 q}{2-q}}\left(B_{s}(x)\right)}\right. \\
& \leq C \epsilon_{0}\|D u\|_{L^{\frac{2 q}{2-q}\left(B_{s}(x)\right)}}
\end{aligned}
$$

This, combined with the Sobolev embedding theorem, implies

$$
\begin{aligned}
\left\|D u_{1}\right\|_{L^{\frac{2 q}{2-q}}\left(B_{\frac{r}{2}}(x)\right)} \leq\left\|D u_{1}\right\|_{L^{\frac{2 q}{2-q}}\left(B_{s}(x)\right)} & \leq C \epsilon_{0}\|D u\|_{L^{\frac{2 q}{2-q}}\left(B_{s}(x)\right)} \\
& \leq C \epsilon_{0}\|D u\|_{L^{\frac{2 q}{2-q}}\left(B_{r}(x)\right)} .
\end{aligned}
$$

Here we have used the fact that $D u_{1}=0$ on $\partial B_{s}(x)$. To estimate $u_{2}$, observe that (1.2) implies that $|T(D u, \cdot)| \leq C|D u| \in L^{4}(\Omega)$ and

$$
\|T(D u, \cdot)\|_{L^{4}(\Omega)} \leq C\|u\|_{W^{2,2}(\Omega)} .
$$

The Moser-Trudinger inequality and (1.2) imply $E^{\prime}(u-\bar{u})-\bar{E}^{\prime}(u-\bar{u}) \in$ $L^{p}(\Omega)$ for any $1<p<\infty$ and

$$
\left\|E^{\prime}(u-\bar{u})-\bar{E}^{\prime}(u-\bar{u})\right\|_{L^{4}(\Omega)} \leq C\|u\|_{W^{2,2}(\Omega)} .
$$

Multiplying (2.4) by $u_{2}$ and integrating it over $B_{s}(x)$, we get

$$
\begin{aligned}
\int_{B_{s}(x)}\left|D^{2} u_{2}\right|^{2} & =\int_{B_{s}(x)}\left|\Delta u_{2}\right|^{2} \\
& \leq C \int_{B_{s}(x)}\left(|D u|\left|D u_{2}\right|+\left|E^{\prime}(u-\bar{u})-\bar{E}^{\prime}(u-\bar{u})\right|\left|u_{2}\right|\right) \\
& \leq C\|u\|_{W^{2,2}(\Omega)}\left(\int_{B_{s}(x)}\left(\left|u_{2}\right|^{2}+\left|D u_{2}\right|^{2}\right)\right)^{\frac{1}{2}} \\
& \leq C\|u\|_{W^{2,2}(\Omega)} r\left(\int_{B_{s}(x)}\left|D^{2} u_{2}\right|^{2}\right)^{\frac{1}{2}} \cdot
\end{aligned}
$$


Here we have applied the Poincaré inequality for $u_{2}$ in the last step. Thus

$$
\int_{B_{s}(x)}\left|D^{2} u_{2}\right|^{2} \leq C\|u\|_{W^{2,2}(\Omega)}^{2} r^{2} .
$$

This, combined with the Sobolev embedding theorem, gives

$$
\int_{B_{s}(x)}\left|D u_{2}\right|^{4} \leq C\left(\int_{B_{s}(x)}\left|D^{2} u_{2}\right|^{2}\right)^{2} \leq C r^{4}\|u\|_{W^{2,2}(\Omega)}^{2} .
$$

In particular, for any $q \in\left(1, \frac{4}{3}\right)$, we have

$$
\left(\frac{r}{2}\right)^{\frac{2 q}{2-q}-4} \int_{B_{\frac{r}{2}}(x)}\left|D u_{2}\right|^{\frac{2 q}{2-q}} \leq C\left(\|u\|_{W^{2,2}(\Omega)}\right) r^{\frac{2 q}{2-q}} .
$$

Since $u_{3}$ is a biharmonic function on $B_{s}(x)$, we know that

$$
\int_{B_{s}(x)}\left|D^{2} u_{3}\right|^{2} \leq \int_{B_{s}(x)}\left|D^{2} u\right|^{2} .
$$

A standard Caccipolli-type argument implies that

$$
\int_{B_{\frac{r}{3}}(x)}\left|D^{2} u_{3}\right|^{2} \leq C r^{-2} \int_{B_{\frac{r}{2}}(x)}\left|D u_{3}\right|^{2} .
$$

This, combined with the subharmonicity of $\left|\Delta u_{3}\right|^{2}$, implies

$$
r^{2}\left\|D u_{3}\right\|_{L^{\infty}\left(B_{\frac{r}{4}}(x)\right)}^{2} \leq C \int_{B_{\frac{r}{3}}(x)}\left|D^{2} u_{3}\right|^{2} \leq C r^{-2} \int_{B_{\frac{r}{2}}(x)}\left|D u_{3}\right|^{2} .
$$

In particular, for any $\theta \in\left(0, \frac{1}{4}\right)$ and $q \in\left(1, \frac{4}{3}\right)$,

$$
(\theta r)^{\frac{2 q}{2-q}-4} \int_{B_{\theta r}(x)}\left|D u_{3}\right|^{\frac{2 q}{2-q}} \leq C \theta^{\frac{2 q}{2-q}} r^{\frac{2 q}{2-q}-4} \int_{B_{\frac{r}{2}}(x)}\left|D u_{3}\right|^{\frac{2 q}{2-q}} .
$$

Putting (2.7), (2.13), (2.15) together, we obtain, for any $q \in\left(1, \frac{4}{3}\right)$ and $\theta \in\left(0, \frac{1}{4}\right)$,

$$
\begin{aligned}
(\theta r)^{\frac{2 q}{2-q}-4} \int_{B_{\theta r}(x)}|D u|^{\frac{2 q}{2-q}} \leq\left(C \epsilon_{0} \theta^{\frac{2 q}{2-q}-4}+\right. & \left.C \theta^{\frac{2 q}{2-q}}\right) r^{\frac{2 q}{2-q}-4} \int_{B_{r}(x)}|D u|^{\frac{2 q}{2-q}} \\
& +C\left(\theta, q,\|u\|_{W^{2,2}(\Omega)}\right) r^{\frac{2 q}{2-q}}
\end{aligned}
$$

Therefore, by choosing $\theta_{0}=(4 C)^{\frac{q-2}{2 q}}$ and then choosing $\epsilon_{0}$ sufficiently small, we have

$$
\begin{aligned}
\left(\theta_{0} r\right)^{\frac{2 q}{2-q}-4} \int_{B_{\theta_{0} r}(x)}|D u|^{\frac{2 q}{2-q}} \leq \frac{1}{2} r^{\frac{2 q}{2-q}-4} \int_{B_{r}(x)}|D u|^{\frac{2 q}{2-q}} & \\
& +C\left(q,\|u\|_{W^{2,2}(\Omega)}\right) r^{\frac{2 q}{2-q}} .
\end{aligned}
$$


Set $p=\frac{2 q}{2-q}$. Observe that $p \in(2,4)$ for $q \in\left(1, \frac{4}{3}\right)$. This completes the proof of Lemma A.

Acknowledgement. I thank Professor S.Y. Alice Chang of the Princeton University Department of Mathematics for informing me of this problem and for helpful discussions on it.

\section{References}

[C] S.Y. Chang, On a fourth-order partial differential equation in conformal geometry, Essays in honor of Alberto P. Calderón. Papers from the International Conference held at the University of Chicago, Chicago, IL, February 1996, (Michael Christ, Carlos E. Kenig and Cora Sadosky, eds.), University of Chicago Press, Chicago, IL, 1999, 127-150, MR 1743859 (2001g:58059), Zbl 0982.53036.

[CGY] S.Y. Chang, M. Gursky and P. Yang, Regularity of a fourth order nonlinear PDE with critical exponent, Amer. J. Math., 121(2) (1999), 215-257, MR 1680337 (2000b:49066), Zbl 0921.35032.

[CWY] S.Y. Chang, L. Wang and P. Yang, A regularity theory of biharmonic maps, Comm. Pure Appl. Math., 52(9) (1999), 1113-1137, MR 1692148 (2000j:58025), Zbl 0953.58013.

[CY] S.Y. Chang and P. Yang, Extremal metrics of zeta function determinants on 4-manifolds, Ann. of Math., 142 (1995), 171-212, MR 1338677 (96e:58034), Zbl 0842.58011.

[SU] R. Schoen and K. Uhlenbeck, A regularity theory for harmonic maps, J. Differential Geom., 17 (1982), 307-335, MR 0664498 (84b:58037a), Zbl 0521.58021.

[UV] K. Uhlenbeck and J. Viaclovsky, Regularity of weak solutions to critical exponent variational equations, Math. Res. Lett., 7(5-6) (2000), 651-656, MR 1809291 (2001j:35059), Zbl 0977.58020.

Received June 20, 2002

Department of Mathematics

UNIVERSITY OF KENTUCKY

LEXINGTON KY 40506

E-mail address: cywang@ms.uky.edu

The author was partially supported by NSF grant DMS 9970549. 